\title{
Sleep disturbances and gastrointestinal dysfunction are associated with thalamic atrophy in Parkinson's disease
}

\author{
Flavia Niccolini ${ }^{1}$ D, Heather Wilson ${ }^{1}$, Beniamino Giordano ${ }^{1}$, Konstantinos Diamantopoulos ${ }^{1}$, Gennaro Pagano',
} Kallol Ray Chaudhuri ${ }^{2}$ and Marios Politis ${ }^{1 *}$

\begin{abstract}
Background: Non-motor symptoms are common aspects of Parkinson's disease (PD) occurring even at the prodromal stage of the disease and greatly affecting the quality of life. Here, we investigated whether non-motor symptoms burden was associated with cortical thickness and subcortical nuclei volume in PD patients.

Methods: We studied 41 non-demented PD patients. Non-motor symptoms burden was assessed using the NonMotor Symptoms Scale grading (NMSS). Cortical thickness and subcortical nuclei volume analyses were carried out using Free-Surfer. PD patients were divided into two groups according to the NMSS grading: mild to moderate (NMSS: $0-40$ ) and severe (NMSS: $\geq 41$ ) non-motor symptoms.

Results: Thalamic atrophy was associated with higher NMSQ and NMSS total scores. The non-motor symptoms that drove this correlation were sleep/fatigue and gastrointestinal tract dysfunction. We also found that PD patients with severe non-motor symptoms had significant thalamic atrophy compared to the group with mild to moderate nonmotor symptoms.

Conclusions: Our findings show that greater non-motor symptom burden is associated with thalamic atrophy in PD. Thalamus plays an important role in processing sensory information including visceral afferent from the gastrointestinal tract and in regulating states of sleep and wakefulness.
\end{abstract}

Keywords: Parkinson's disease, Non-motor symptoms, Cortical thickness, Thalamus, MRI

\section{Background}

Non-motor symptoms have been recognised as an important component of Parkinson's disease (PD). They occur in almost all the PD patients [1-3] and can precede the onset of motor symptoms $[4,5]$. Sleep disturbances, depression, gastrointestinal and urinary dysfunction, fatigue and cognitive impairment are often unresponsive to dopamine replacement therapy and greatly affect the quality of life of PD patients [6].

Structural Magnetic Resonance (MR) imaging studies have used T1-weighted MRI together with automated

\footnotetext{
*Correspondence: marios.politis@kcl.ac.uk

${ }^{1}$ Neurodegeneration Imaging Group, Institute of Psychiatry, Psychology and Neuroscience (loPPN), King's College London, London, UK

Full list of author information is available at the end of the article
}

computerised methods such as voxel-based morphometry (VBM) and cortical thickness measures to assess the morphology of cortical gray matter. Both morphometric analysis methods have been widely used to investigate neuroanatomical correlates of neurological disorders.

Previous MR imaging studies have shown cortical and subcortical changes in PD patients with cognitive impairment [7-12]. Mood disorders were also associated with cortical and subcortical changes in PD patients [13, 14]. Surface based morphometric analysis showed that depressed PD patients had significant increased cortical thickness in the orbitofrontal regions and insula compared to non-depressed PD patients [14] and loos of volume of the left nucleus accumbens was found in PD patients with apathy [13]. Significant grey matter volume loss in the lingual gyrus and superior parietal lobe was 
found in PD patients with visual hallucinations compared to healthy controls and PD patients without visual hallucinations [15]. Rapid eye movement (REM) behavior disorders (RBD) in PD patients was associated to atrophy of the pontomesencephalic tegmentum, medullary reticular formation, hypothalamus, thalamus, putamen, amygdala and anterior cingulate cortex [16]. Additionally, other symptoms, which can occur as non-motor manifestations of PD were associated with cortical and subcortical volumetric changes. Voxel-based morphometric analysis showed that patients with idiopathic restless leg syndrome (RLS) had significant loss of grey matter in the bilateral primary somatosensory cortex, which additionally extended into left-sided primary motor areas [17]. Voxel-based morphometric analysis showed loss of volumes in the left occipital cortex and parahippocampal gyrus of patients with chronic fatigue syndrome [18]. Volumetric changes were also observed in the right insula of patients with neurocardiogenic syncope suggesting that insular dysfunction may play a role in the development of neurocardiovascular symptoms [19].

Functional MR imaging (fMRI) studies have also shown abnormal functional communication and activation between brain regions, which play a role in the pathophysiology of non-motor symptoms in PD [20]. Dysfunction of the posterior cingulate cortex during executive task was linked to cognitive impairment in PD [21]. A research study using resting state fMRI (rs-fMRI) showed that frontal, postcentral, and anterior cingulate cortex regions are involved in fatigue in PD patients [22]. Other functional imaging studies demonstrated the involvement of both dorsal and ventral visual pathways in the pathogenesis of visual hallucinations in PD, [23]. Functional MR imaging provides a powerful tool to understand human brain organization. However, fMRI is complicated by several factors such as the fact that functional coupling changes dynamically, suggesting that it is constrained by, but not fully dictated by, anatomic connectivity. Moreover, fMRI is sensitive to head motion and to differences in the mental states of participants during the scans. Morphological MR imaging studies might provide a more objective insight in the pathophysiology of PD non-motor symptoms.

Clinical assessment of the overall burden grading of non-motor symptoms using validated questionnaires and scales is now possible [24, 25], however, the neural substrate of non-motor symptoms burden remain unclear. Previous studies using neuroimaging techniques such as positron emission tomography (PET) and quantitative susceptibility mapping (QSM) MRI have shown lack of association between non-motor symptoms burden as measured by Non-motor Symptoms Scale for PD (NMSS) and dopaminergic deficits and iron accumulation [26,
27]. In this study, we aimed for the first time to investigate whether non-motor symptoms burden is associated with cortical and subcortical morphological changes in a cohort of non-demented PD patients.

\section{Materials and methods \\ Participants and clinical characteristics}

Forty-one patients with a diagnosis of idiopathic PD according to the Queen Square Brain Bank criteria were recruited from specialist Movement Disorders clinics at King's College Hospital, London (Table 1). None of these patients fulfilled the diagnostic criteria of PD mild cognitive impairment or PD dementia [28, 29]. All participants screened successfully to undertake MRI scanning under scanning standard criteria (http://www.mrisafety.com) and had no history of other neurological or psychiatric disorders.

NMSS and the patient self-reported Non-motor Symptoms Questionnaire (NMSQ) were used to assess non-motor symptoms. The NMSS is a healthcare professional-completed scale measuring the severity (mild, moderate, and severe) and frequency (every day, several times a week, once a week, and rarely) of 30 different NMS divided into 9 distinct domains [25]. Sleep disturbances includes four questions that assess excessive daytime sleepiness, fatigue, insomnia and RLS thus providing a comprehensive measure of daytime and nocturnal sleep disturbances in PD.

Neuropsychiatric symptoms were assessed with the Beck Depression Inventory-II (BDI-II), and the Hamilton Depression Rating Scale (HDRS). The Mini Mental Status Examination (MMSE) was used to assess general cognitive status. Motor symptom burden was assessed with

\section{Table 1 Clinical characteristics of Parkinson's disease patients}

PD patients

\begin{tabular}{ll}
\hline No $(M, \%)$ & $41(24,58.5 \%)$ \\
Age (years; median \pm SD) & $65.0 \pm 9.3$ \\
Disease duration ${ }^{a}$ (months; median \pm SD) & $84.0 \pm 71.8$ \\
Daily LED (mg; median \pm SD) & $480.0 \pm 957.7$ \\
H\&Y (median \pm SD) & $2.0 \pm 1.0$ \\
NMSS (median \pm SD) & $37.0 \pm 31.4$ \\
NMSS Domain 2: sleep/fatigue (median \pm SD) & $4.0 \pm 7.7$ \\
NMS Domain 6: gastrointestinal tract (median $\pm S D)$ & $3.0 \pm 6.0$ \\
NMSQ (median $\pm S D)$ & $8.0 \pm 5.9$ \\
MMSE (median $\pm S D)$ & $30.0 \pm 1.3$ \\
PDQ-39 [(median $\pm S D) \pm S D]$ & $27.5 \pm 24.4$ \\
\hline
\end{tabular}

H\&Y Hoein \& Yahr, LED levodopa equivalent dose, MMSE mini mental status examination, NMSS Non-motor Symptoms Scale, NMSQ Non-motor Symptoms Questionnaire, $P D Q-39$ 39-item Parkinson's disease Questionnaire

a From time of first appearance of Parkinson's disease motor symptoms 
the Hoehn \& Yahr (H\&Y) staging and it was performed OFF medication after overnight withdrawal of patient's dopaminergic medications. Quality of life was measured with the patient self-reported 39-item PD Questionnaire (PDQ-39). Daily dopaminergic medication dose (LED) was calculated with a formula based on the theoretical equivalence to levodopa [28].

PD patients were divided into two groups according to the non-motor symptoms burden as assessed by the NMSS [25]: (1) PD patients with mild to moderate (NMSS levels 0-2, NMSS scores: 0-40; No: 23) and (2) severe (NMSS levels 3 and 4, NMSS scores: $\geq 41$; No: 18) non-motor symptoms burden (Table 2).

The study was approved by the institutional review boards and the Surrey Borders research ethics committee. Written informed consent was obtained from all study participants in accordance with the Declaration of Helsinki.

\section{Imaging data analysis}

MRI scans were acquired with a 32-channel head coil on a Siemens Magneton Trio Trim syngo MR B17 (Erlangen, Germany) 3-Tesla MRI scanners. A T1-weighted three-dimensional magnetization-prepared rapid acquisition gradient echo [MPRAGE; time repetition $(\mathrm{TR})=2300 \mathrm{~ms}$, time echo $(\mathrm{TE})=2.98 \mathrm{~ms}$, flip angle of $9^{\circ}$, time to inversion $(\mathrm{TI})=900 \mathrm{~ms}$, matrix $=240 \times 256$; voxel size $=1 \mathrm{~mm}$ ] was acquired. MRI scans were performed OFF medication after overnight withdrawal of patient's dopaminergic medications to avoid motion

Table 2 Clinical characteristics of Parkinson's disease patients with mild to moderate and severe non-motor symptoms burden

\begin{tabular}{lll}
\hline & $\begin{array}{l}\text { PD mild } \\
\text { to moderate } \\
\text { NMS burden } \\
\text { (NMSS }=\mathbf{0 - 4 0 )}\end{array}$ & $\begin{array}{l}\text { PD severe } \\
\text { NMS burden } \\
\text { (NMSS } \geq \text { 41) }\end{array}$ \\
\hline No (\%) & $23(56.1 \%)$ & $18(43.9 \%)$ \\
Gender (M, \%) & $15(65.2 \%)$ & $9(50 \%)$ \\
Age (years; median \pm SD) & $58.5 \pm 8.9$ & $70.3 \pm 8.3^{*}$ \\
Disease duration ${ }^{\text {a }}$ (months; & $16.1 \pm 60.4$ & $108.0 \pm 73.8^{*}$ \\
median \pm SD) & & \\
Daily LED (mg; median \pm SD) & $250.3 \pm 438.3$ & $690.0 \pm 1219.6^{*}$ \\
H\&Y OFF (median \pm SD) & $1.0 \pm 0.8$ & $2.3 \pm 1.2^{*}$ \\
MMSE (median \pm SD) & $30.0 \pm 1.6$ & $29.5 \pm 1.3$ \\
PDQ-39 (median \pm SD) & $9.5 \pm 16.1$ & $47.0 \pm 24.4^{* * *}$ \\
\hline
\end{tabular}

H\&Y Hoehn \& Yahr, LED levodopa equivalent dose, MMSE mini mental status examination, NMSS Non-motor Symptoms Scale, PDQ-39 39-item Parkinson's disease Questionnaire, UPDRS-III Unified Parkinson's Disease Rating Scale part III ${ }^{*} P<0.05 ;{ }^{* * *} P<0.001$

a From time of first appearance of Parkinson's disease motor symptoms artefact induced by motor complications such as levodopa-induced dyskinesia.

\section{FreeSurfer analysis}

FreeSurfer's image analysis suite (version 5.3.0 http:// surfer.nmr.mgh.harvard.edu) was used to process individual MRI scans for deriving region-of-interest (ROI)based cortical thickness and subcortical volume analysis. Briefly, the whole-brain T1-weighted images underwent a correction for intensity homogeneity, skull strip, and segmentation into grey and white matter with intensity gradient and connectivity among voxels [30]. Cortical thickness was measured as the distance from the grey/ white matter boundary to the corresponding pial surface [31]. Reconstructed data sets were visually inspected for accuracy, and segmentation errors were corrected. Subcortical nuclei volumes were derived by automated procedures, which assign a neuroanatomical label to each voxel in an MRI volume based on probabilistic information automatically estimated from a manually labelled training set [32]. All individual volumes were normalized for intracranial volume (ICV) automatically generated by FreeSurfer [33]. Since no laterality was observed and for minimizing the number of comparisons, average hemispheric $C T h$ and subcortical nuclei values were processed in the statistical analysis.

\section{Voxel-wise volumetric analysis}

Voxel-wise volumetric analysis was carried out using SPM12 implemented in Matlab 2015a [34]. T1 weighted MR images were spatially normalized to the T1 MNI template. MRI images were smoothed by application of $8 \mathrm{~mm}$ full-width at half maximum Gaussian kernel. Z-score maps were derived on a voxel basis using the general linear model. Subgroup analysis was carried out between PD patients with mild to moderate vs. severe non-motor symptoms (Fig. 2a), PD patients with sleep/ fatigue disturbances vs. those without sleep/fatigue (Fig. 2b) and PD patients with gastrointestinal tract dysfunction vs. those without gastrointestinal tract dysfunction (Fig. 2c). The threshold for statistical significance was set to $P<0.05$ cluster-wise corrected.

\section{Statistical analysis}

Statistical analysis and graph illustration were performed with SPSS (version 20 Chicago, Illinois, USA) and GraphPad Prism (version 6.0c) for MAC OS X, respectively. For all variables, variance homogeneity and Gaussianity were tested with Shapiro-Wilk test. A sample size of $40 \mathrm{PD}$ patients was adequate to detect significant correlations between MRI values and clinical measures of 0.79 with $80 \%$ power using $1 \%$ type I error. We recruited 41 PD patients to account for an approximate 5-10\% 
drop-out. We interrogated correlations between ROIbased FreeSurfer's volume analysis and clinical data using Spearman's $r$ correlation coefficient and we applied the Benjamini-Hochberg correction. Multivariate analysis of variance (MANOVA) was used to assess morphological differences in cortical thickness and subcortical nuclei volumes derived by FreeSurfer's analysis between PD patients with mild to moderate and severe non-motor symptoms burden. If the overall multivariate test was significant, $P$ values for each variable were calculated following Benjamini-Hochberg multiple-comparisons test in order to reduce false discovery rate. We set the false discovery rate cut-off at 0.05 . Subsequently, MANOVA was repeated adding disease duration and daily LED as covariate since both disease duration and levodopa treatment have been associated to a cortical thinning [8, 35, 36]. PD patients were then subdivided in two groups according to the presence of sleep disturbances (NMSS subdomain 2; 29 PD patients with sleep disturbances) and GI dysfunction (NMSS subdomain 6; 23 PD patients with GI dysfunction). All data are presented as mean \pm SD, and the level of $\alpha$ was set for all comparisons at $P<0.05$, Benjamini-Hochberg corrected. For voxel-wise statistics appropriately weighted contrasts were used to derive $\mathrm{Z}$-scores on a voxel basis using the general linear model; threshold for statistical significant was set to $P<0.05$.

\section{Results}

We found that loss of thalamic volume was associated to higher NMSQ $(r=-0.42, P=0.042)$ and NMSS $(r=-0.47, P=0.014)$ total scores (Fig. 1a). Within the NMSS subscores sleep/fatigue $(r=-0.36, P=0.042)$ and gastrointestinal tract dysfunction $(r=-0.36, P=0.042)$ were the non-motor symptoms that drove this correlation (Fig. 1b). No associations were found between nonmotor symptoms burden and other subcortical nuclei volumes or cortical thickness (all $P>0.10$ ).

\section{Structural changes in the group of PD patients with mild to moderate and severe non-motor disease burden}

PD patients with severe non-motor symptoms burden were older $(P=0.024)$, had significant longer disease duration $(P=0.014)$, were receiving higher amounts of daily LED $(P=0.024)$ and had higher H\&Y scores $(P=0.014)$ than those with mild to moderate non-motor symptoms burden (Table 2). PD patients with severe non-motor symptoms burden had also worse quality of life scores than those with mild to moderate non-motor disease burden $(P<0.001)$. PD patients with severe nonmotor symptoms burden had higher NMSS Domain 2: Sleep/fatigue subscores compared to those with mild to moderate non-motor symptoms burden $(8.68 \pm 8.7$ vs $2.17 \pm 3.5 ; P<0.001)$. In specific, PD patients with severe non-motor symptoms burden had higher scores at Subdomain 5: Does the patient have difficulties falling or staying asleep? compared to those with mild to moderate non-motor symptoms burden $(2.42 \pm 1.4$ vs $0.87 \pm 1.7$; $P<0.01)$.

We found significant differences in subcortical nuclei volumes between the groups of PD patients with mild to moderate and severe non-motor symptoms ( $F$ $(9,21)=1.16 ; P=0.045$; Table 3$)$. PD patients with severe non-motor symptoms burden had significant loss of thalamic volume compared to the group with mild to moderate non-motor symptoms burden $(P=0.048)$. There were no differences in caudate, putamen, globus pallidus, nucleus accumbens, amygdala and hippocampus between the two groups. Since PD patients with severe non-motor symptoms burden had longer disease duration, higher $\mathrm{H} \& \mathrm{Y}$ scores and were on higher daily LED, we repeated the multivariate analysis adding disease duration, H\&Y and daily LED as covariate and this did not influence the results. Whole brain voxel-wise analysis between the group of PD patients with mild to moderate and severe non-motor symptoms confirmed the results from Freesurfer's analysis. Whole brain analysis revealed clusters of significant volume loss in PD patients with severe non-motor symptoms in the thalamus $(P<0.05$; Fig. 2a).

We then subdivided the PD patients into two groups according to the presence of sleep/fatigue disturbances and gastrointestinal tract dysfunction. PD patients with sleep/fatigue disturbances showed significant thalamic atrophy compared those without sleep/fatigue disturbances $(P=0.033$; Fig. 2b). Significant thalamic volume loss was also observed in PD patients with gastrointestinal tract dysfunction compared those without gastrointestinal tract dysfunction ( $P=0.043$; Fig. 2c).

No significant differences in cortical thickness were found between the two groups (all $P>0.10$ ). Vertex-wise analysis also did not show any significant differences in cortical areas between the PD patients with mild to moderate and severe non-motor symptoms burden.

\section{Discussion}

Our findings indicate that higher non-motor symptoms burden is associated with thalamic atrophy in PD patients and in specific sleep/fatigue disorders and gastro-intestinal dysfunction were the non-motor symptoms, which drove this correlation. It is unclear why thalamic atrophy has been associated only to two nonmotor symptoms. One possible explanation is that the thalamus plays a key role in modulating the flow of sensory and motor information to and from the cerebral 

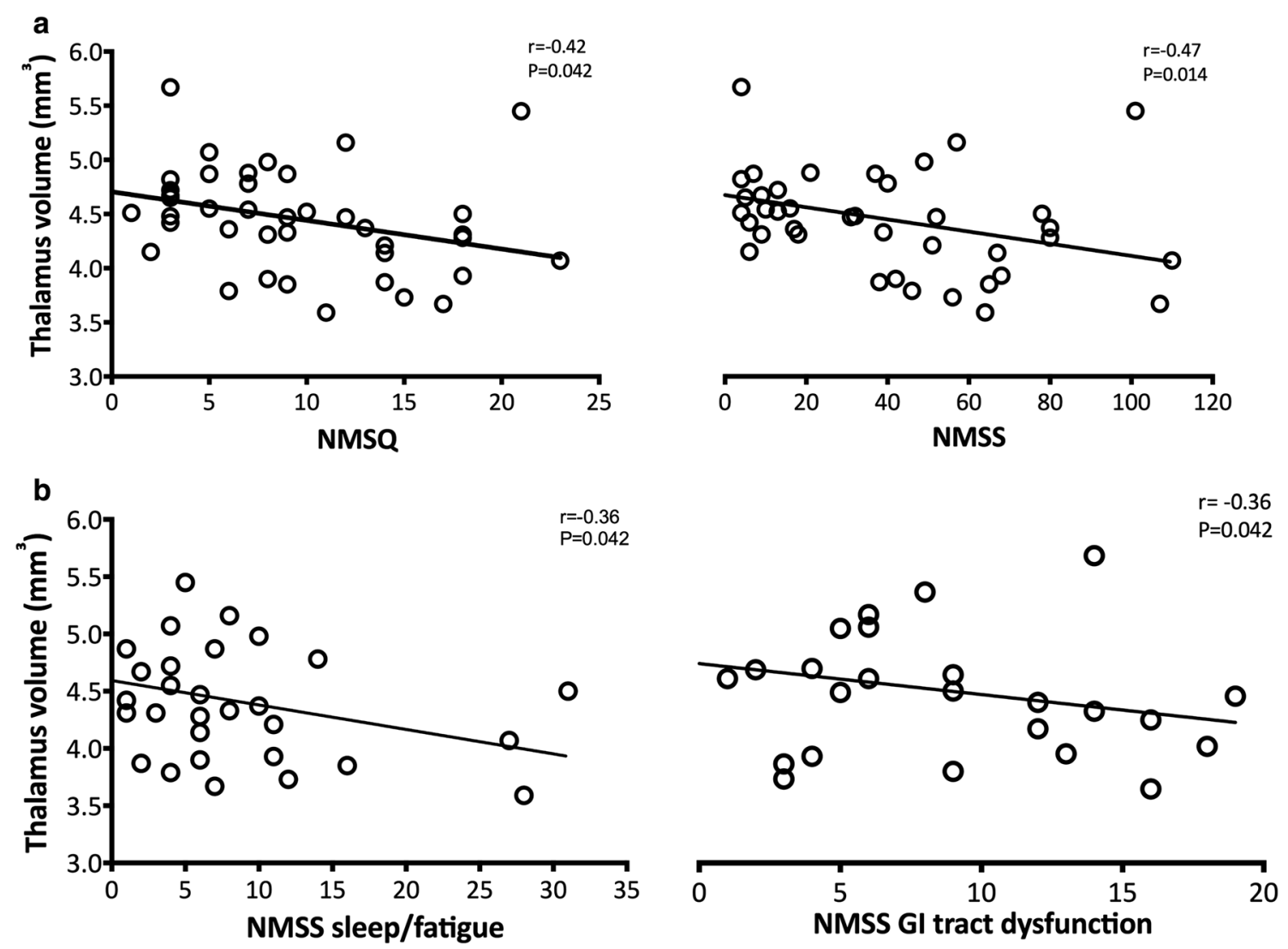

Fig. 1 Correlations between thalamic atrophy and non-motor symptoms in Parkinson's disease patients. Loss of thalamic volume correlated with a higher NMSQ $(r=-0.42, P=0.042)$ and NMSS $(r=-0.47, P=0.014)$ total scores; $\boldsymbol{b}$ higher NMSS domain 2 sleep $/$ fatigue $(r=-0.36, P=0.042)$ and NMSS domain 6 gastrointestinal tract dysfunction $(r=-0.36, P=0.042)$ subscores

Table 3 Subcortical nuclei volume in Parkinson's disease patients with mild to moderate and severe to very severe nonmotor symptoms burden

\begin{tabular}{lllcc}
\hline & $\begin{array}{l}\text { PD mild to moderate NMS burden } \\
\text { (NMSS = 0-40) }\end{array}$ & $\begin{array}{l}\text { PD severe NMS burden } \\
\text { (NMSS } \geq \text { 41) }\end{array}$ & P value* & \% change \\
\hline Caudate (median \pm SD) & $2.30 \pm 0.2$ & $2.40 \pm 0.3$ & $>0.10$ & +2.5 \\
Putamen (median \pm SD) & $3.30 \pm 0.3$ & $3.20 \pm 0.3$ & $>0.10$ & -3.6 \\
Globus Pallidus (median \pm SD) & $1.00 \pm 0.2$ & $1.00 \pm 0.1$ & $>0.10$ & -9.1 \\
Thalamus (median \pm SD) & $4.60 \pm 0.4$ & $4.20 \pm 0.5$ & 0.048 & -9.1 \\
Hippocampus (median \pm SD) & $2.50 \pm 0.3$ & $2.40 \pm 0.3$ & $>0.10$ & -7.7 \\
Amygdala (median \pm SD) & $1.00 \pm 0.1$ & $0.96 \pm 0.1$ & 0.06 & -11.3 \\
Nucleus accumbens (median \pm SD) & $0.30 \pm 0.1$ & $0.28 \pm 0.1$ & 0.06 & -9.1 \\
\hline
\end{tabular}

Italic values indicate the significant result $P<0.05$

* $P$ values are Benjamini-Hochberg corrected for multiple comparisons

cortex thus regulating the sleep/wake cycle pathway and gastrointestinal function.

While, morphological studies earlier have shown thalamic atrophy in PD patients, this is the first study showing a significant association between thalamic atrophy and non-motor symptoms. Non-motor symptom burden grading is currently the only validated clinical system to assess the overall impact of non-motor symptoms [25]. In clinical practice, this is important as many studies, which address non-motor symptoms may do so in a piecemeal fashion, addressing selected non-motor symptoms such as cognition 


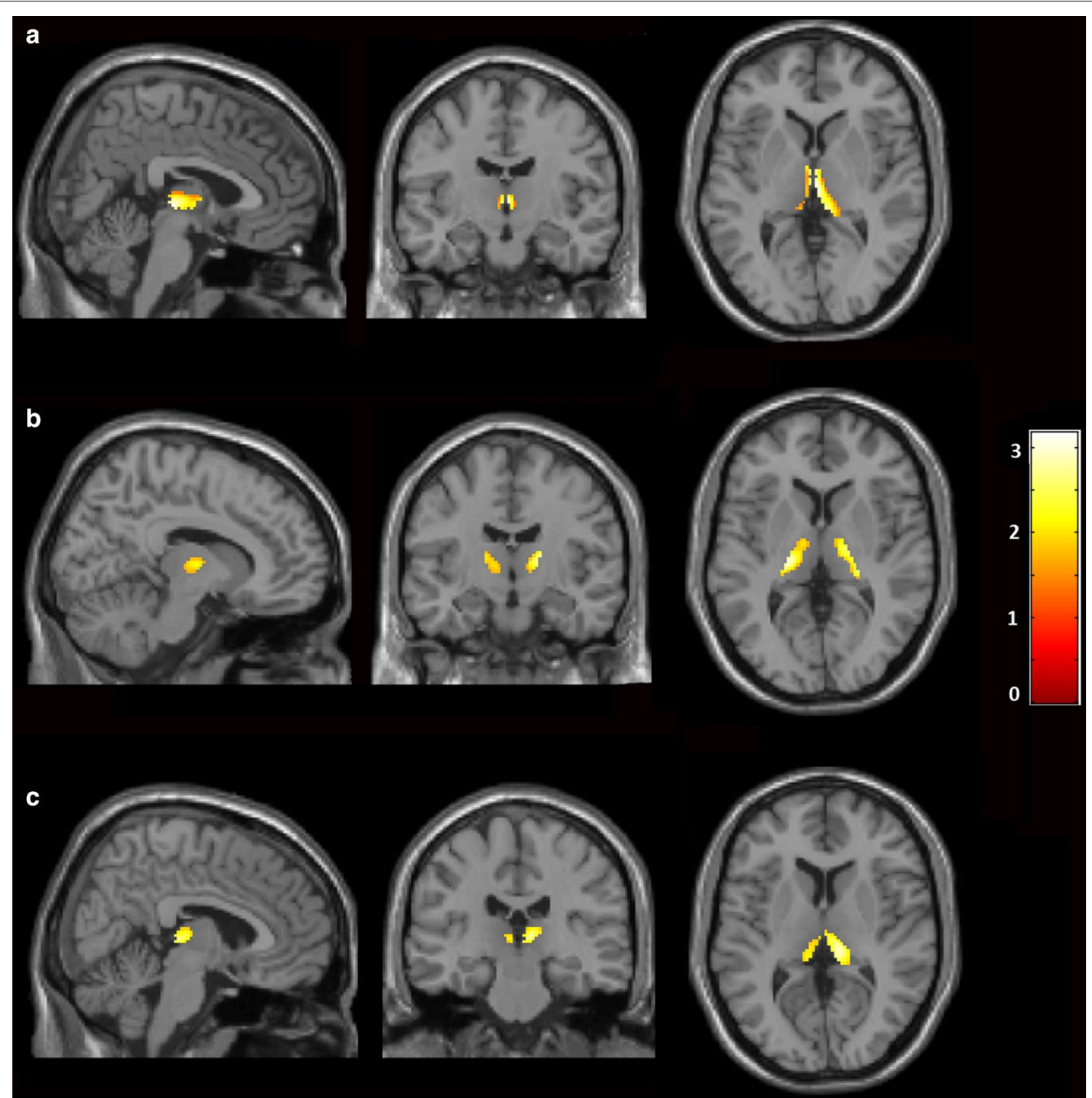

Fig. 2 Thalamic atrophy in Parkinson's disease patients. Statistical parametric maps of a PD patients with mild to moderate vs. severe non-motor symptoms; b PD patients with sleep/fatigue disturbances vs. those without sleep/fatigue; c PD patients with gastrointestinal tract dysfunction vs. those without gastrointestinal tract dysfunction. Yellow-red areas represent voxel clusters of significant volume loss. The colour stripe indicates $z$-values. MNI co-ordinates: $\mathbf{a} x=3.85, y=-12.23, z=3.37 ; \mathbf{b} x=11.55, y=-11.46, z=4.90 ; \mathbf{c} x=9.24, y=-26.85, z=7.22$

rather than the overall burden. Studies have also shown that non-motor symptoms burden, rather than individual non-motor symptoms may be a key determinant of quality of life in PD [37]. Based on this classification system, when we divided our cohort of PD patients into two groups according to the severity of non-motor symptoms, we found that PD patients with severe nonmotor symptoms burden had $9 \%$ significant loss of thalamic volume compared to those with mild to moderate non-motor-symptoms burden.
We found significant correlation between worse sleep/ fatigue disturbances and thalamic atrophy in our cohort of PD patients. Sleep disturbances are one of the most common non-motor symptoms in PD occurring in up to $90 \%$ of PD patients [37-39]. Sleep disturbances may precede by many years the classic motor abnormalities of $\mathrm{PD}^{33}$ and their frequency increases with the progression of the disease $[40,41]$. Sleep disturbances in PD can be divided in daytime manifestation, such as excessive daytime sleepiness, and nocturnal disturbances, 
which include insomnia, obstructive apnoea, restless legs syndrome (RLS) and rapid eye movement (REM) sleep behaviour disorder (RBD) [37].

The thalamus modulates the flow of sensory and motor information to and from the cerebral cortex and plays an important role in regulating the sleep/wake cycle pathway [42]. In PD, loss of dopamine in mesocorticolimbic pathway targeting the thalamus causes sleep/wake cycle dysfunction leading to disorders of thalamocortical arousal with lack of normal thalamocortical rhythms during night-time and excessive sleepiness during daytime $[43,44]$. The role of thalamus in sleep disturbances is supported by preclinical and clinical data that showed lesions of the thalamus in cats and bilateral thalamic lesions in humans cause severe and persistent insomnia $[45,46]$. Moreover, neuron loss was observed in the thalamus of patients with familial and sporadic fatal insomnia [47, 48]. Liu et al. [48] have shown loss of regional grey matter in the bilateral thalamus of 12 healthy subjects following $72 \mathrm{~h}$ sleep deprivation suggesting a close link between sleep disturbance and thalamus volume. Resting state functional MR imaging studies have shown reduced spontaneous activity in the thalamus of subjects with insomnia, which was associated with early morning awakening suggesting that hypoactivity of the thalamus may lead to an increased transmission of sensory signals from the ascending reticular activating system which cause awakening [48]. Thalamocortical functional connectivity was also decreased in 14 healthy volunteers following $36 \mathrm{~h}$ of sleep deprivation [49] and impaired thalamocortical connectivity is also involved in RLS symptoms [50, 51]. Thus, thalamic atrophy may account for the sleep/disturbances observed in PD.

Thalamic atrophy was also associated with worse gastrointestinal symptoms in our cohort of PD patients. The gastrointestinal tract is affected earlier in the course of PD and could play an important role in the pathogenesis of the disease providing a site of initiation for the accumulation and propagation of $\alpha$-synuclein [52]. Gastrointestinal dysfunction in PD encompasses several symptoms ranging from oral issues such as drooling and swallowing difficulties to gastroparesis and constipation [53]. The thalamus receives signals from the gastrointestinal tract through the spinal or vagal afferents and projects to cortical areas, which process visceral sensation [54]. Previous MR imaging studies have shown decreased grey matter density in the thalamus of patients with irritable bowel symptoms $[55,56]$. Moreover, white matter changes encompassing lower fractional anisotropy and increased mean diffusivity were observed in the basal ganglia and thalamus of patients with irritable bowel symptoms suggesting that patients with chronic gastrointestinal disorders may display microstructural changes within the cortico-basal ganglia-thalamic loop [57]. One of the symptoms assessed by the NMSS domain 6 is the difficulty in swallowing, functional MR imaging has shown that the thalamus, specifically the ventral posterior lateral nucleus, is involved in the oral-sensory stimulation and cerebral control of swallowing [58]. Our findings suggest that thalamus may play a role in the development of gastrointestinal dysfunction in PD.

Although PD patients with severe non-motor symptoms had longer disease duration, higher H\&Y scores and were on higher daily LED, we did not find any effect of disease severity and dopaminergic treatment on the association between non-motor symptoms and thalamic atrophy. Whereas cortical grey matter atrophy has been shown to be related to disease duration, basal ganglia and in specific striatal atrophy was not correlated with duration of the disease [59]. Moreover, a recent study investigating non-motor symptoms burden in a large cohort of PD patients has shown high prevalence of non-motor symptoms even at the early-untreated stage of the disease, thus suggesting that the non-motor symptoms burden is independent from disease severity or dopaminergic treatment [60]. PD patients with severe non-motor symptoms had also worse quality of life scores than those with mild to moderate non-motor symptoms. This is in line with previous studies showing that non-motor symptoms have a greater impact on quality of life than motor symptoms and is common even at the early stage of the disease [61, 62].

A limitation of this study is the lack of an age-sex matched control group, which could have clarified whether thalamic atrophy is a distinct feature of PD unrelated to non-motor symptoms burden. Previous morphological studies using a control group have shown thalamic atrophy in PD patients with and without mild cognitive impairment, which was associated to worse cognitive impairment at 18-month follow-up [63].

\section{Conclusions}

This is the first study showing an association between higher non-motor symptom burden and thalamic atrophy in PD. Among the non-motor symptoms, sleep/fatigue disturbances and gastrointestinal dysfunction were the non-motor symptoms that drove this correlation. Further studies, investigating morphological brain changes and non-motor symptoms burden in a larger cohort of PD patients and using specific scale to assess night and day time sleep disturbances such as the PD Sleep Scale and Epworth Sleep Scale and instrumental assessments for GI 


\section{dysfunction such as endoscopic evaluation of swallowing, are needed to confirm our findings.}

\author{
Abbreviations \\ LED: levodopa equivalent dose; MRI: Magnetic Resonance Imaging; NMSQ: \\ Non-motor Symptom Questionnaire; NMSS: Non-motor Symptom Scale; PDQ-
} 39: 39-item Parkinson's disease Questionnaire.

\section{Acknowledgements}

We thank all participants and their families, the MRI radiographers, and the clinical research nurses at Imanova Ltd for their cooperation and support with this study.

\section{Authors' contributions}

MP conceived the study, conceptualized the experimental design and acquired funding for the study. FN gave input to experimental design. FN and GP performed the imaging and clinical assessments and acquired the data. FN, and GP recruited the subjects. FN, GP and MP organised the study. FN wrote the first draft and prepared the manuscript. FN generated the figures. HW analysed the data. FN, MP, BG, KD and KRC interpreted the data. All authors revised and gave input to the manuscript. All authors read and approved the final manuscript.

\section{Funding}

This research did not receive any specific grant from funding agencies in the public, commercial, or not-for-profit sectors.

\section{Availability of data and materials}

Clinical and MRI data used to support the findings of this study are restricted by the Surrey Borders research ethics committee in order to protect patient privacy. Data are available from Dr Flavia Niccolini, Maurice Wohl Clinical Neuroscience Institute

Institute of Psychiatry, Psychology \& Neuroscience, King's College London, 125 Coldharbour Lane, Camberwell, London SE5 9NU, UK, email: flavia.niccolini@ kcl.ac.uk, 0044-(0)-207-848-5755 for researchers who meet the criteria for access to confidential data.

\section{Ethics approval and consent to participate}

The study was approved by the Surrey Borders research ethics committee. Written informed consent was obtained from all study participants in accordance with the Declaration of Helsinki.

\section{Consent for publication}

Participants have expressed their consent for anonymized data publication in written or verbal form.

\section{Competing interests}

The authors declare that they have no competing interests.

\begin{abstract}
Author details
${ }^{1}$ Neurodegeneration Imaging Group, Institute of Psychiatry, Psychology and Neuroscience (IoPPN), King's College London, London, UK. ${ }^{2}$ National Parkinson Foundation International Centre of Excellence, Department of Basic \& Clinical Neuroscience, King's College London and Kings College Hospital, London, UK.
\end{abstract}

Received: 14 April 2019 Accepted: 12 October 2019

Published online: 22 October 2019

\section{References}

1. Martinez-Martin P, Schapira AHV, Stocchi F, et al. Prevalence of non-motor symptoms in Parkinson's disease in an international setting; study using non-motor symptoms questionnaire in 545 patients. Mov Disord. 2007;22:1623-9.

2. Krishnan S, Sarma G, Sarma S, Kishore A. Do nonmotor symptoms in Parkinson's disease differ from normal aging? Mov Disord. 2011;26:2110-3.
3. Kim HS, Cheon SM, Seo JW, Ryu HJ, Park KW, Kim JW. Nonmotor symptoms more closely related to Parkinson's disease: comparison with normal elderly. J Neurol Sci. 2013;324:70-3.

4. Chaudhuri KR, Healy D, Schapira AHV. The non-motor symptoms of PD disease. Diagn Manag Lancet Neurol. 2006:5:235-45.

5. Berg D, Postuma RB, Bloem B, et al. Time to redefine PD? Introductory statement of the MDS task force on the definition of Parkinson's disease. Mov Disord. 2014;29:454-62.

6. Hely MA, Morris JGL, Reid WGJ, Traficante R. Sydney multicenter study of Parkinson's disease: non-L-dopa responsive problems dominate at 15 years. Mov Disord. 2005;20:190-9.

7. Hanganu A, Bedetti C, Jubault T, et al. Mild cognitive impairment in patients with Parkinson's disease is associated with increased cortical degeneration. Mov Disord. 2013;28:1360-9.

8. Hanganu A, Bedetti C, Degroot C, et al. Mild cognitive impairment is linked with faster rate of cortical thinning in patients with Parkinson's disease longitudinally. Brain. 2014;137:1120-9.

9. Pagonabarraga J, Corcuera-Solano I, Vives-Gilabert Y, et al. Pattern of regional cortical thinning associated with cognitive deterioration in Parkinson's disease. PLoS ONE. 2013;8:e54980.

10. Garcia-Diaz Al, Segura B, Baggio HC, et al. Structural MRI correlates of the MMSE and pentagon copying test in Parkinson's disease. Parkinsonism Relat Disord. 2014;20(12):1405-10.

11. Pereira JB, Svenningsson $P$, Weintraub D, et al. Initial cognitive decline is associated with cortical thinning in early Parkinson disease. Neurology. 2014;82:2017-25.

12. Segura B, Baggio HC, Marti MJ, et al. Cortical thinning associated with mild cognitive impairment in Parkinson's disease. Mov Disord. 2014;29(12):1495-503.

13. Carriere N, Besson P, Dujardin K, et al. Apathy in Parkinson's disease is associated with nucleus accumbens atrophy: a magnetic resonance imaging shape analysis. Mov Disord. 2014;29(7):897-903.

14. Huang P, Lou Y, Xuan M, et al. Cortical abnormalities in Parkinson's disease patients and relationship to depression: a surface-based morphometry study. Psychiatry Res. 2016;250:24-8.

15. Ramírez-Ruiz B, Martí MJ, Tolosa E, et al. Cerebral atrophy in Parkinson's disease patients with visual hallucinations. Eur J Neurol. 2007;14(7):750-6.

16. Boucetta S, Salimi A, Dadar M, Jones BE, Collins DL, Dang-Vu TT. Structural brain alterations associated with rapid eye movement sleep behavior disorder in Parkinson's disease. Sci Rep. 2016;6:26782.

17. Unrath A, Juengling FD, Schork M, Kassubek J. Cortical grey matter alterations in idiopathic restless legs syndrome: an optimized voxel-based morphometry study. Mov Disord. 2007;22(12):1751-6.

18. Puri BK, Jakeman PM, Agour M, et al. Regional grey and white matter volumetric changes in myalgic encephalomyelitis (chronic fatigue syndrome): a voxel-based morphometry $3 \mathrm{~T} \mathrm{MRI}$ study. Br J Radiol. 2012;85:e270-3.

19. Kim JB, Suh SI, Seo WK, Koh SB, Kim JH. Right insular atrophy in neurocardiogenic syncope: a volumetric MRI study. AJNR Am J Neuroradiol. 2014;35:113-8.

20. Prell T. Structural and functional brain patterns of non-motor syndromes in Parkinson's disease. Front Neurol. 2018;12(9):138.

21. Fransson P, Marrelec $G$. The precuneus/posterior cingulate cortex plays a pivotal role in the default mode network: evidence from a partial correlation network analysis. Neuroimage. 2008;42(3):1178-84.

22. Li J, Yuan $Y$, Wang $M$, Zhang J, Zhang L, Jiang S, et al. Alterations in regional homogeneity of resting-state brain activity in fatigue of Parkinson's disease. J Neural Transm. 2017;124(10):1187-95.

23. Yao N, Shek-Kwan Chang R, Cheung C, Pang S, Lau KK, Suckling J, et al. The default mode network is disrupted in Parkinson's disease with visual hallucinations. Hum Brain Mapp. 2014;35(11):5658-66.

24. Chaudhuri KR, Martinez-Martin P, Sherman R, et al. The non-motor staging of Parkinson's disease: results from an international pilot study. Neurology. 2009;72(Suppl 3):A322.

25. Chaudhuri KR, Rojo JM, Schapira AHV, et al. A proposal for a comprehensive grading of Parkinson's disease severity combining motor and nonmotor assessments: meeting an unmet need. PLoS ONE. 2013;8:e57221.

26. Park SB, Kwon KY, Lee JY, et al. Lack of association between dopamine transporter loss and non-motor symptoms in patients with Parkinson's disease: a detailed PET analysis of 12 striatal subregions. Neurol Sci. 2019:40(2):311-7. 
27. Shin C, Lee S, Lee JY, Rhim JH, Park SW. Non-motor symptom burdens are not associated with iron accumulation in early Parkinson's disease: a quantitative susceptibility mapping study. J Korean Med Sci. 2018;33(13):e96.

28. Politis M, Wu K, Loane C, et al. Staging of serotonergic dysfunction in Parkinson's disease: an in vivo 11C-DASB PET study. Neurobiol Dis. 2010;40:216-21.

29. Litvan I, Aarsland D, Adler CH, et al. MDS Task Force on mild cognitive impairment in Parkinson's disease: critical review of PD-MCI. Mov Disord. 2011;26:1814-24

30. Fischl B, Sereno MI, Dale AM. Cortical surface-based analysis. II: Inflation, flattening, and a surface-based coordinate system. Neuroimage. 1999;9(2):195-207.

31. Fischl B, Dale AM. Measuring the thickness of the human cerebral cortex from magnetic resonance images. Proc Natl Acad Sci USA. 2000;97:11050-5

32. Fischl B, Salat DH, Busa E, et al. Whole brain segmentation: automated labeling of neuroanatomical structures in the human brain. Neuron. 2002;33:341-55.

33. Malone IB, Leung KK, Clegg S, et al. Accurate automatic estimation of total intracranial volume: a nuisance variable with less nuisance. Neuroimage. 2015;104:366-72.

34. Friston KJ, Holmes AP, Worsley KJ, Poline JB, Frith CD, Frackowiak RS. Statistical parametric maps in functional imaging: a general linear approach. Hum Brain Mapp. 1995;2:189-210.

35. Uribe C, Segura B, Baggio HC, Abos A, Garcia-Diaz Al, Campabadal A, et al. Progression of Parkinson's disease patients'subtypes based on cortical thinning: 4-year follow-up. Parkinsonism Relat Disord. 2019;64:286-92.

36. Tessitore A, Santangelo G, De Micco R, Vitale C, Giordano A, Raimo S, et al. Cortical thickness changes in patients with Parkinson's disease and impulse control disorders. Parkinsonism Relat Disord. 2016;24:119-25.

37. Comella CL. Sleep disorders in Parkinson's disease: an overview. Mov Disord. 2007;22(Suppl. 17):S367-73.

38. Shyamal HM, Morgan JC, Sethi KD. Sleep disorders associated with Parkinson's disease: role of dopamine, epidemiology and clinical scales of assessment. CNS Spectr. 2008;13(Suppl. 4):6-11.

39. Politis M, Wu K, Molloy S, Bain GP, Chaudhuri KR, Piccini P. Parkinson's disease symptoms: the patient's perspective. Mov Disord. 2010;25(11):1646-51.

40. dos Santos AB, Kohlmeier KA, Barreto GE. Are sleep disturbances preclinical markers of Parkinson's disease? Neurochem Res. 2015;40(3):421-7.

41. Zoccolella S, Savarese M, Lamberti P, Manni R, Pacchetti C, Logroscino G. Sleep disorders and the natural history of Parkinson's disease: the contribution of epidemiological studies. Sleep Med Rev. 2011;15(1):41-50.

42. Jan JE, Reiter RJ, Wasdell MB, Bax M. The role of the thalamus in sleep, pineal melatonin production, and circadian rhythm sleep disorders. J Pineal Res. 2009;46(1):1-7.

43. Rye DB. The two faces of Eve: dopamine's modulation of wakefulness and sleep. Neurology. 2004;63(Suppl 3):S2-7.

44. Rye DB, Bliwise DL, Dihenia B, Gurecki P. Daytime sleepiness in Parkinson's disease. J Sleep Res. 2000;9:63-9.

45. Lugaresi E. The thalamus and insomnia. Neurology. 1992;42(7 Suppl 6):28-33.

46. Quigg M, Frysinger RC, Harrison M, Elias WJ. Acute insomnia following surgery of the ventralis intermedius nucleus of the thalamus for tremor. J Clin Sleep Med. 2007;3(1):58-9.
47. Montagna P. Fatal familial insomnia and the role of the thalamus in sleep regulation. Handb Clin Neurol. 2011;99:981-96.

48. Liu CH, Liu CZ, Zhang J, et al. Reduced spontaneous neuronal activity in the insular cortex and thalamus in healthy adults with insomnia symptoms. Brain Res. 2016;1648:317-24.

49. Shao $Y$, Wang $L$, Ye E, et al. Decreased thalamocortical functional connectivity after 36 hours of total sleep deprivation: evidence from resting state FMRI. PLOS ONE. 2013;8(10):e78830.

50. Ku J, Cho YW, Lee YS, et al. Functional connectivity alternation of the thalamus in restless legs syndrome patients during the asymptomatic period: a resting-state connectivity study using functional magnetic resonance imaging. Sleep Med. 2014;15(3):289-94.

51. Gorges M, Rosskopf J, Müller HP, Lindemann K, Hornyak M, Kassubek J. Patterns of increased intrinsic functional connectivity in patients with restless legs syndrome are associated with attentional control of sensory inputs. Neurosci Lett. 2016;617:264-9.

52. Braak H, Rub U, Gai WP, Del Tredici K. Idiopathic Parkinson's disease: possible routes by which vulnerable neuronal types may be subject to neuroinvasion by an unknown pathogen. J Neural Transm. 2003;110:517-36.

53. Fasano A, Visanji NP, Liu LW, Lang AE, Pfeiffer RF. Gastrointestinal dysfunction in Parkinson's disease. Lancet Neurol. 2015;14(6):625-39.

54. Aziz Q, Schnitzler A, Enck P. Functional neuroimaging of visceral sensation. J Clin Neurophysiol. 2000;17:604-12.

55. Davies KD, Pope G, Chen J, Kwan CL, Crawley AP, Diamant NE. Cortical thinning in IBS: implications for homeostatic, attention, and pain processing. Neurology. 2008;70:153-4.

56. Seminowicz DA, Labus JS, Bueller JA, et al. Regional gray matter density changes in brains of patients with irritable bowel syndrome. Gastroenterology. 2010;139(1):48-57.

57. Ellingson BM, Mayer E, Harris RJ, et al. Diffusion tensor imaging detects microstructural reorganization in the brain associated with chronic irritable bowel syndrome. Pain. 2013;154(9):1528-41.

58. Lowell SY, Poletto CJ, Knorr-Chung BR, Reynolds RC, Simonyan K, Ludlow $\mathrm{CL}$. Sensory stimulation activates both motor and sensory components of the swallowing system. Neuroimage. 2008;42(1):285-95.

59. Lewis MM, Du G, Lee EY, Nasralah Z, Sterling NW, Zhang L, et al. The pattern of gray matter atrophy in Parkinson's disease differs in cortical and subcortical regions. J Neurol. 2016;263(1):68-75.

60. Zis P, Martinez-Martin P, Sauerbier A, et al. Non-motor symptoms burden in treated and untreated early Parkinson's disease patients: argument for non-motor subtypes. Eur J Neurol. 2015;22(8):1145-50.

61. Martinez-Martin P, Rodriguez-Blazquez C, Kurtis MM, Chaudhuri KR, NMSS Validation Group. The impact of non-motor symptoms on healthrelated quality of life of patients with Parkinson's disease. Mov Disord. 2011;26(3):399-406.

62. Vasconcellos LF, Pereira JS, Adachi M, Greca D, Cruz M, Malak AL, Charchat-Fichman H. Volumetric brain analysis as a predictor of a worse cognitive outcome in Parkinson's disease. J Psychiatr Res. 2018;102:254-60.

63. Foo H, Mak E, Yong TT, Wen MC, Chander RJ, Au WL, Sitoh YY, Tan LC, Kandiah N. Progression of subcortical atrophy in mild Parkinson's disease and its impact on cognition. Eur J Neurol. 2017;24(2):341-8.

\section{Publisher's Note}

Springer Nature remains neutral with regard to jurisdictional claims in published maps and institutional affiliations. 